e-Polymers 2001, no. 006.

\title{
PVC/poly(norbornene ester) blends: mixtures of flexible and rigid random coils
}

\author{
Florian Raubacher, Mark D. Roth, Joachim H. Wendorff*
}

Fachbereich Chemie, Institut für Physikalische Chemie, Kernchemie und

Makromolekulare Chemie und Wissenschaftliches Zentrum für

Materialwissenschaften, Hans-Meerwein-Straße, Philipps-Universität Marburg,

D-35043 Marburg, Germany; Fax 0049-6421-28-28916;

wendorff@mailer.uni-marburg.de

(Received: May 30, 2001; published: July 11, 2001)

\begin{abstract}
Considered are blends of the flexible coil-shaped poly(vinyl chloride) (PVC) and rigid coil-shaped polymers, poly(norbornene ester)s, characterized by strong rotational constraints. The unexpected finding is that the stepwise increase of the specific heat at the glass transition $T_{\mathrm{g}}$ as well as the relaxation of the enthalpy in a temperature interval below $T_{\mathrm{g}}$ (physical aging) vanish as the concentration of poly(norbornene ester)s increases in blends with PVC. The interpretation is that the very specific nature of the glass transition of the rotationally confined poly(norbornene ester) - cooperative librations within the minima of the rotational potential - controls the relaxation properties of the blends. The composition vs. glass transition temperature relation is strongly curved for the blends due to the very small increase of the specific heat of the poly(norbornene ester)s at the glass transition.
\end{abstract}

\section{Introduction}

Polynorbornene and its derivatives such as the poly(norbornene ester)s poly(norbornene-2-carboxylic acid methyl ester) (PNME) and poly(norbornene-2carboxylic acid ethyl ester) (PNEE), see Scheme 1, are bicyclic polyolefins. The bulky monomer units which are located along the chain backbone give rise to strong rotational constraints: the rotational barriers are much larger than the thermal energy $k T$ even at temperatures well above room temperature [1-5]. These constraints give rise to unique conformational and dynamic features. 


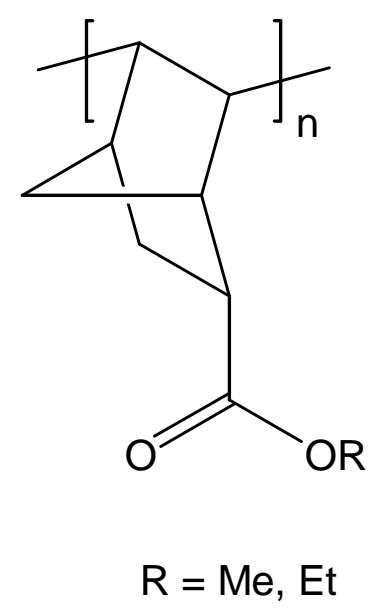

Scheme 1. Poly(norbornene carboxylic acid ester)s. $R=$ Me: PNME; $R=E:$ PNEE

The chain molecules display, on the one hand, a random Gaussian coil conformation very similar to the case of flexible chain molecules such as poly(methyl methacrylate) or poly(vinyl chloride) $[2,6,7]$. This is obvious from extended computer simulations based on force field approaches as well as from light scattering studies and viscometry performed on solutions $[6,7]$. Yet computer simulations show that the Gaussian coil chain molecules considered here are rigid: no conformational changes originating from torsional jumps across rotational barriers seem to be possible in solutions and in the molten state up to temperatures approaching decomposition. This strong chain rigidity is apparently responsible for a set of interesting properties.

One is that the chain packing within the glassy state is extremely dense as judged from the ratio $R$ of the real volume taken by the monomer units $V_{\text {real }}$ to the hard core Van der Waals volume $V_{\mathrm{VdW}}$.

$R=V_{\text {real }} / V_{\text {VdW }}$

The value of $R$ found for the polynorbornenes amounts to 1.43 and thus corresponds to the average value found for flexible chain molecules in the crystalline state [8]. The glassy state of flexible chain molecules, on the other hand, is characterized by an average value of 1.55 . Positron annihilation studies have revealed furthermore that the fractional free volume of polynorbornene and polynorbornene derivatives is much smaller than the corresponding values found for flexible chain molecules [9]. The peculiar gas permeation properties reported for the polynorbornenes have been attributed to this dense molecular packing and the low value of the fractional free volume [9].

It is obvious that the dense packing and the strong rotational constraints will also influence molecular relaxations both in the glassy state and at the transition into the molten state. In fact, studies on the composition dependence of the glass transition temperatures of polynorbornene-based copolymers such as norbornene-5hexyl/norbornene copolymers [10] as well as thermomechanical investigations have revealed the presence of a glass transition for various polynorbornenes. They are located at temperatures at which no torsional jumps across rotational barriers are expected from the predictions of computer simulations. The interpretation is that the glass transition has to originate from motions other than the torsional jumps usually associated with glass relaxation processes. We have tentatively assigned it to cooperative librational motions within the minima of the rotational potential, as 
proposed in theoretical papers by Moro $[11,12]$. Molecular dynamics calculations support this interpretation.

Another support comes from the surprising experimental finding that the increase of the specific heat at the glass transition is so small that it cannot be resolved by standard DSC-techniques. We conclude that the glass transition has very unique features. Penetration studies involving the penetration of a stylus as a function of the temperature in the neighborhood of $T_{\mathrm{g}}$ also reveal that the dynamic state above the glass transition temperature differs from the one experienced by flexible chain molecules. The stylus penetrates only a short distance at the glass transition temperature and does not continue to penetrate as the temperature is further increased, in contrast to the case of flexible chain molecules.

The results described above in detail picture a class of bicyclic polyolefines with very unusual conformational and dynamic properties which might be of interest for technical applications such as membranes, protective coatings etc. The disadvantage of the polynorbornene and the esters is that they are very brittle even at high molecular weights beyond $10^{6} \mathrm{~g} / \mathrm{mol}$. One way around this problem consists in considering polynorbornene-based copolymers and the other in considering polymer blends. It thus seemed interesting to us to study mixtures of flexible chain molecules and rigid chain polynorbornenes. The concept is that the polynorbornenes will impose some of their peculiar properties on the flexible chain polymers and that, for this reason, such mixtures may be of technical interest. One of the features which will be discussed in this contribution is the variation of the glass transition temperature with the composition, bearing in mind the specific nature of the polynorbornene glass transition temperature and the vanishing increase of the specific heat at the glass transition. A second feature is the physical aging process taking place on annealing within the glassy state [13-16]. Here we expect surprises due to the dense packing and chain rigidity of the polynorbornenes.

\section{Experimental part}

The synthesis of the poly(norbornene carboxylic acid ester)s was described elsewhere [17]. The PVC used was standard PVC "Vestolit" (Chemische Werke Hüls AG, $M_{v}=61000$ ), purified by dissolving in tetrahydrofuran (THF, abs., Fluka) and precipitating from methanol (> $98 \%$, distilled, BASF).

The polymer blends were prepared by dissolving calculated amounts of each component jointly in the solvent THF, evaporizing the solvent and drying the samples in vacuo at $150^{\circ} \mathrm{C}$ for one hour. The absence of solvent was controlled by thermogravimetric measurements. Differential scanning calorimetry was performed on a Mettler DSC-30 with heating and cooling rates of $10 \mathrm{~K} / \mathrm{min}$. Midpoint transition temperatures of the second heating runs were used to determine the glass transition temperatures. The specific heat increments were calculated from the extrapolated step heights at the midpoint transition temperatures.

\section{Results and discussion}

\section{Compatibility of the poly(norbornene ester)s with flexible chain polymers}

One problem which had to be addressed was the compatibility of rigid and flexible chains. Rigid rod-like molecules are known to display a strong tendency towards incompatibility with flexible chain molecules due to entropic effects [18]. Rigid chain 
molecules such as polynorbornene and poly(norbornene ester)s should not display such a tendency due to their coil-shaped conformation. Investigations on ternary solutions of polynorbornene and poly(norbornene ester)s with various flexible chain polymers such as PMMA, PS or poly(4-chlorostyrene) in different solvents gave indications that the unmodified polynorbornene is an unfavourable partner as far as blending with the flexible chain polymers mentioned above is concerned, whereas the poly(norbornene ester)s seem to be very favourable partners with respect to poly(vinyl chloride) [6]. It is for this reason that we concentrated on blends of poly(norbornene ester)s with PVC. The blends were prepared from solutions using tetrahydrofuran as solvent.

\section{Composition dependence of the glass transition}

\section{Pure components}

To begin with we will consider calorimetric results of the pure components in the temperature range of the glass transition. PVC is characterized by a stepwise increase of the specific heat as the glass transition temperature is approached. The increase amounts to about $0.28 \mathrm{~J} /(\mathrm{g} \cdot \mathrm{K})$, a value which is well documented in the literature and which can easily be resolved experimentally [19]. On the other hand, we were not able to detect any sign of a glass transition for the poly(norbornene ester)s as far as calorimetric investigations are concerned. This is in agreement with reports in the literature [9] and with similar findings from us for the unmodified polynorbornene. No increase was detected in the temperature range where other methods such as small angle scattering results on density fluctuations revealed the transition. It thus seems that the increase of the specific heat at the glass transition is extremely small or absent for polymers which are characterized by strong rotational confinements. In fact, this observation is not unexpected in view of the peculiar nature of the glass transition discussed above.

The disadvantage is that we currently do not have reliable experimental results on the location of the glass transition temperatures for the poly(norbornene ester)s. In any case they have to be higher than about $250^{\circ} \mathrm{C}$ since the films are still brittle at this temperature and since extrapolations based on copolymers suggest even higher temperatures. We decided to obtain an estimate based on computer simulations.

It has been shown for different polymer systems, among others by Rigby [19] for polyethylene and Soldera [20] for poly(methyl methacrylate), that temperature dependent volumetric properties of amorphous polymers can successfully be simulated by molecular dynamics simulation: We therefore used this approach to get an estimate on the location of the glass transition temperature of the poly(norbornene carboxylic acid methyl ester) and to get an impression on the peculiar character of this glass transition. These simulations were performed with the Cerius2 software of $\mathrm{MSI}$. In order to model the high rotational barriers a new force field for polynorbornenes based on the parameters of a standard force field was developed [7]. The intrinsic torsional term of the DREIDING 2.2.1 forcefield [21] was optimized with respect to the results of ab initio calculated rotational potentials of norbornene dimers. Atomic charges were obtained by fitting the quantum mechanically derived electrostatic potential of trimers. The fitting procedure was accomplished as implemented in the RESP program [22]. The molecular dynamics simulations were performed in the isothermal-isobaric NPT-ensemble [23] using periodic boundary conditions of amorphous cells. The integration time step was 1 fs. An RIS-model [24] developed from the torsional energy hypersurfaces of oligomers was used to prepare 
the initial starting structure consisting of a parent chain of 50 monomers. The geometry optimization with subsequent annealing generated a relaxed structure. The system was cooled down from $850 \mathrm{~K}$ to $300 \mathrm{~K}$ in $50 \mathrm{~K}$-steps each with an equilibration time of $100 \mathrm{ps}$ followed by a 100 ps sampling run in which the volume was profiled as a function of time. The results are shown in Fig. 1.

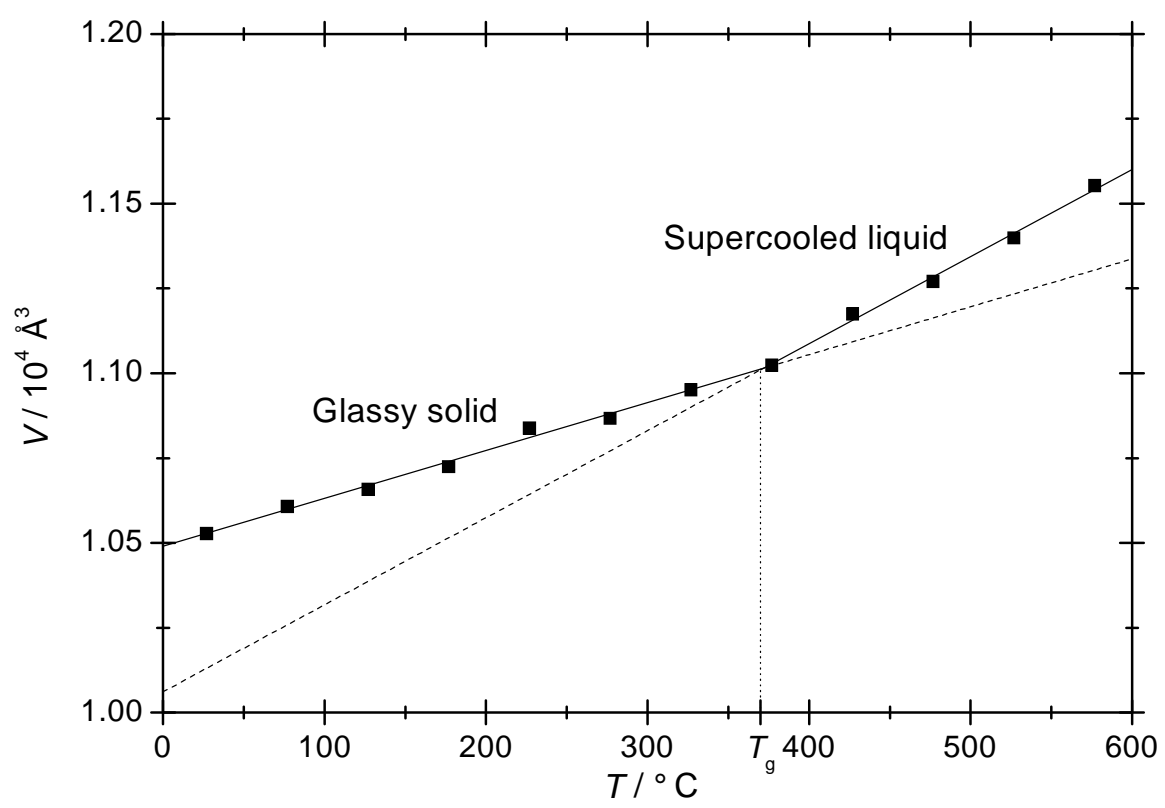

Fig. 1. Volume-temperature curve for poly(norbornene carboxylic acid methyl ester) as obtained by molecular dynamic simulation

The volume-temperature curve given in Fig. 1 shows a distinct change in slope at $370^{\circ} \mathrm{C}$. This change can be interpreted as the signature of the glasstransition. We will use this value in the following discussions.

Blends of poly(norbornene ester)s and poly(vinyl chloride)

Next we will discuss the calorimetric results obtained for the blends of PVC and the poly(norbornene carboxylic acid alkyl ester)s PNME and PNEE. The DSC traces obtained for various compositions of the blends are depicted in Fig. 2 . It is obvious that only one glass transition is obtained for the blends and that the glass transition shifts as a function of the composition. Similar results have been reported for compatible polymer blends and it has been argued that a single glass transition indicates homogeneous mixing on a molecular scale. So we draw the same conclusion here. The blends are optically transparent and optical microscopy does not give indications of a phase transition.

Fig. 2 and Tab. 1 nevertheless display a rather unexpected result. We observe that the magnitude of the stepwise increase of the specific heat at the glass transition decreases with increasing concentration of the poly(norbornene ester)s. An extrapolation indicates that the stepwise increase vanishes as the concentration of the poly(norbornene ester) becomes larger than $80 \mathrm{wt} .-\%$. One conclusion based both on the observation on the pure poly(norbornene ester)s and on the extrapolation 


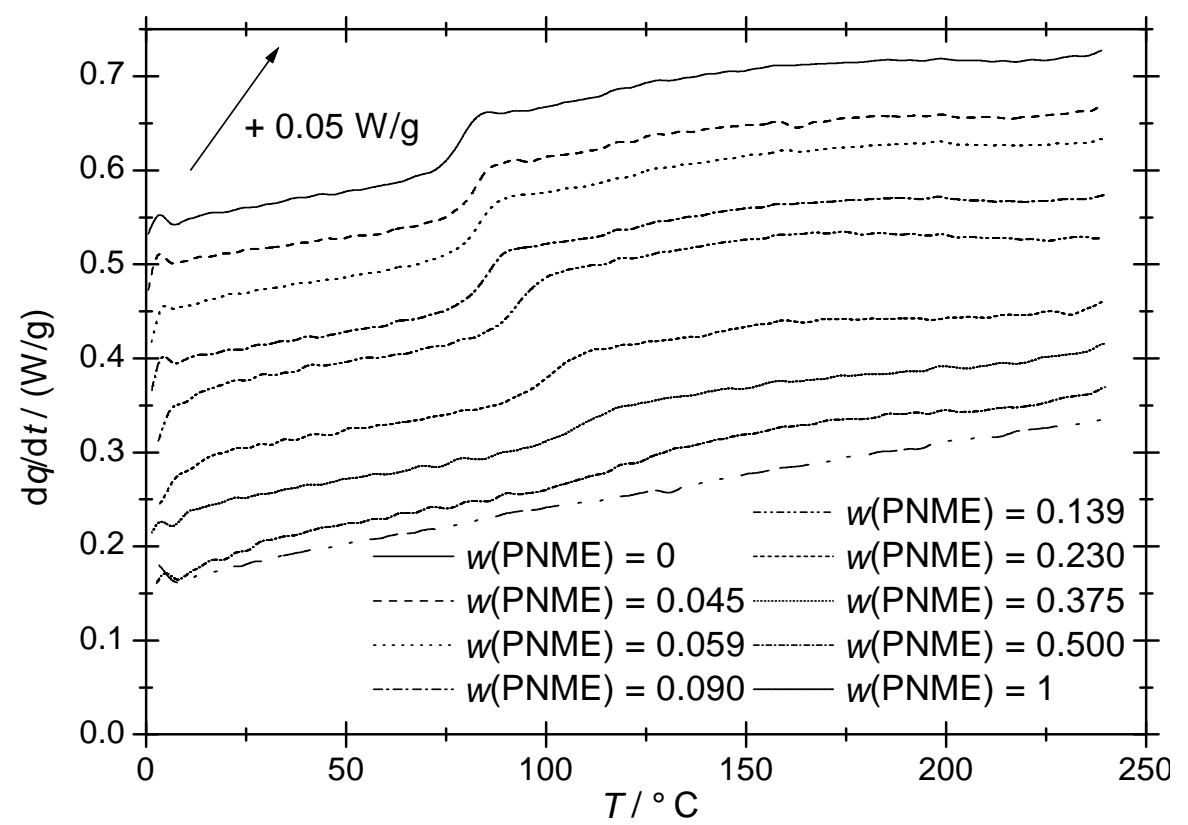

a)

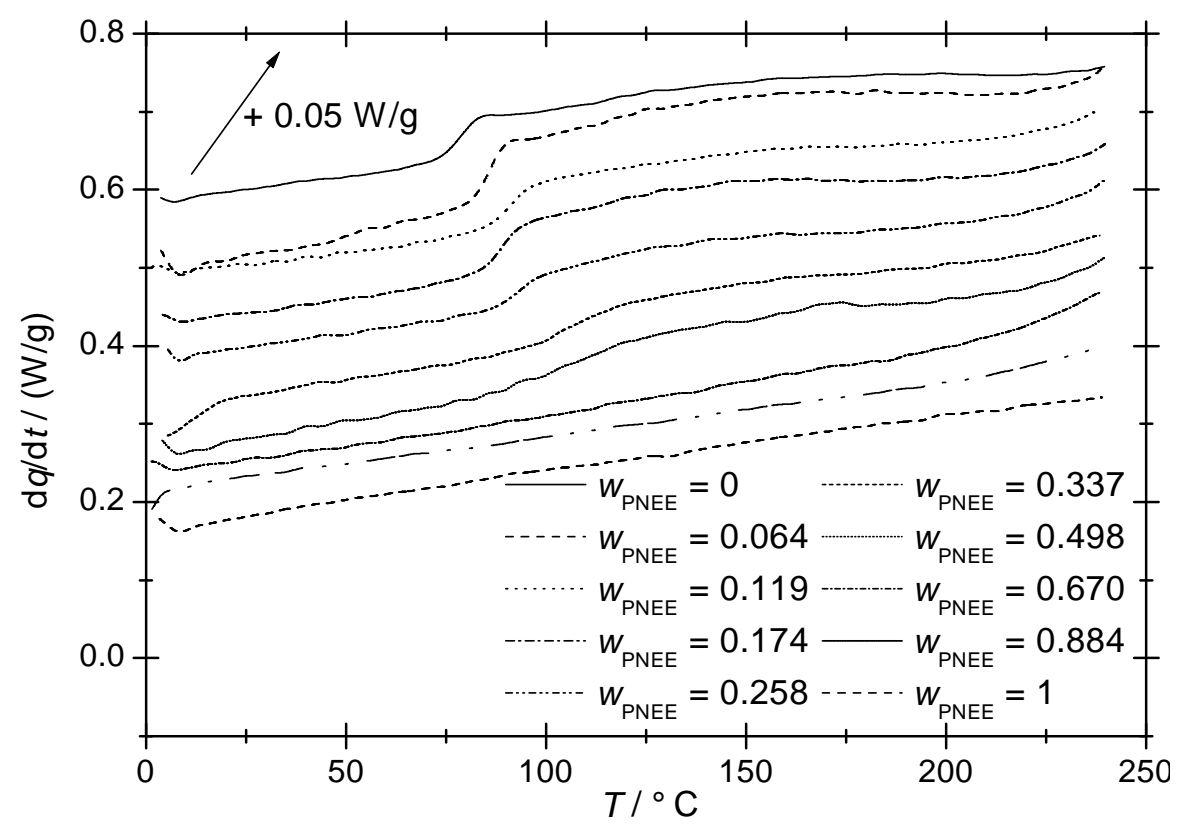

Fig. 2. a) DSC thermograms of PNME-PVC blends. b) DSC thermograms of PNEEPVC blends. (Curves have been plotted with a relative offset of $0.05 \mathrm{~W} / \mathrm{g}$ between each curve from below to above)

is that the poly(norbornene ester)s apparently are characterized by a freezing-in process which happens without any detectable jump of the specific heat. In fact, we were not able to detect a glass transition by calorimetry for polynorbornene, as already discussed above, even though other methods showed the occurrence of a glass transition. It seems that this behavior arises from the special character of the glass transition suggested by the results of the computer simulations on unsubstituted polynorbornene: the rate of torsional transitions is very low, even well above the glass transition temperature. This lack of torsional degrees of freedom obviously accounts for the observed small increment of the heat capacity at the glass transition. We have to conclude from our results that the character of the glass 
transition seems to be different from the one usually found for polymers not only in the case of the polynorbornene derivatives but also in blends containing still relatively large amounts of PVC. The rigid coil molecules seem to impose their dynamic properties on the flexible chain molecules.

Tab. 1. a) Thermal properties of PNME-PVC blends

\begin{tabular}{lcc}
$w($ PNEE $)$ & $T_{\mathrm{g}} /{ }^{\circ} \mathrm{C}$ & $\Delta c_{\mathrm{p}} /\left(\mathrm{J} \cdot \mathrm{g}^{-1} \mathrm{~K}^{-1}\right)$ \\
\hline 0 & 78 & 0.28 \\
0.045 & 82 & 0.29 \\
0.059 & 83 & 0.27 \\
0.090 & 84 & 0.29 \\
0.139 & 92 & 0.25 \\
0.230 & 101 & 0.24 \\
0.375 & 111 & 0.27 \\
0.500 & 127 & 0.19 \\
1 & $--^{*}$ & -
\end{tabular}

Tab. 1. b) Thermal properties of PNEE-PVC blends

\begin{tabular}{lcc}
\hline$w(\mathrm{PNEE})$ & $T_{\mathrm{g}} /{ }^{\circ} \mathrm{C}$ & $\Delta c_{\mathrm{p}} /\left(\mathrm{J} \cdot \mathrm{g}^{-1} \mathrm{~K}^{-1}\right)$ \\
\hline 0 & 78 & 0.28 \\
0.064 & 86 & 0.27 \\
0.119 & 91 & 0.27 \\
0.174 & 89 & 0.26 \\
0.258 & 99 & 0.23 \\
0.337 & 107 & 0.13 \\
0.498 & 110 & 0.19 \\
0.670 & $-^{*}$ & $-^{*}$ \\
0.884 & $-^{*}$ & $-^{*}$ \\
1 & $--^{*}$ & - \\
Not determinable. & &
\end{tabular}

The calorimetric results reflect an additional surprising fact. The glass transition is shifted to higher temperatures as the poly(norbornene ester) is added to PVC as expected in view of the high $T_{\mathrm{g}}$ of the esters. Yet, the results are nevertheless unexpected as apparent from the plot on the composition dependence of the glass transition temperature as displayed in Fig. 3 . The increase of $T_{\mathrm{g}}$ with increasing 
concentration of the poly(norbornene ester) seems to be far too small in view of the high glass transition temperature of the ester.

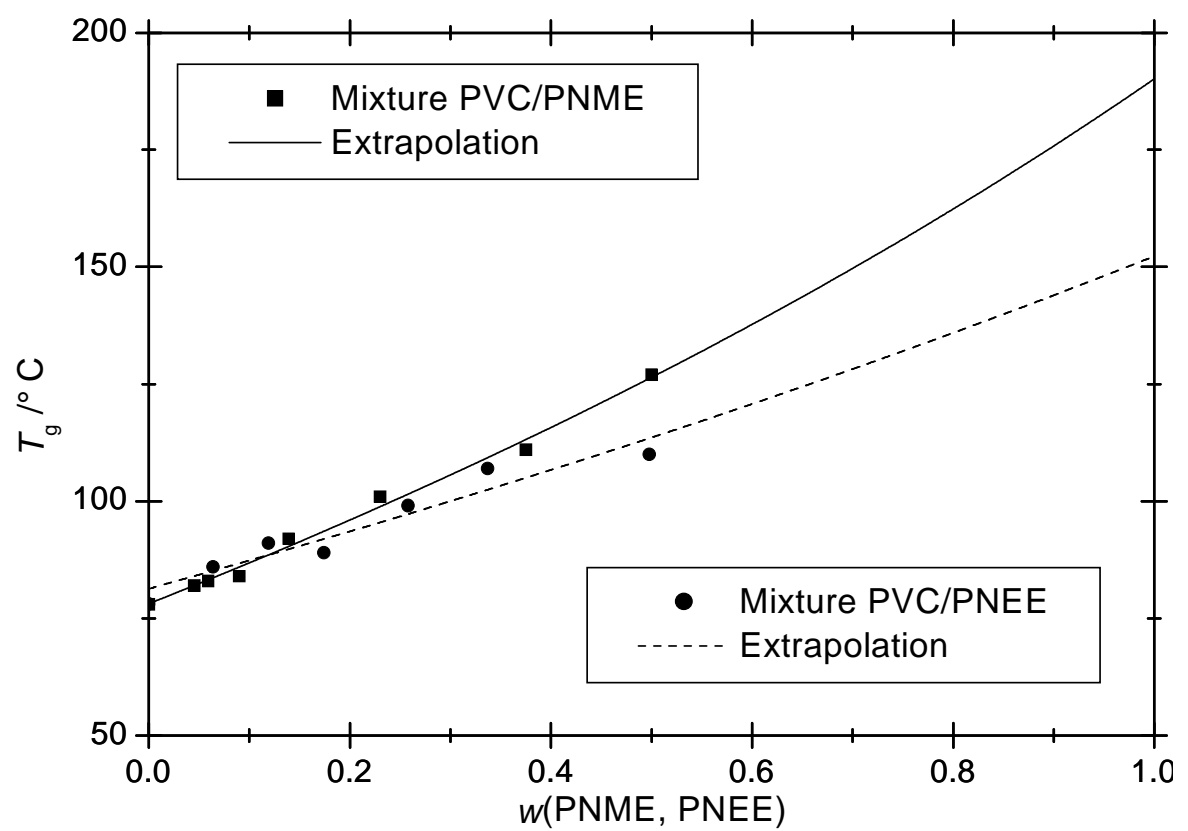

Fig. 3. Glass transition temperatures of PNME-PVC blends and PNEE-PVC blends. Extrapolations according to the Fox equation [25]

The composition dependence of the glass transition temperature has to be more complex than expected on the basis of simple nearly linear relations such as predicted by the Fox equation [25]. This result is not really surprising. This becomes apparent if we use the more general approach by Gordon et al. or Couchman et al. [26-28]. Their approach is based on considerations of the continuity of extensive thermodynamic parameters such as the entropy at the glass transition. They derived, for instance, an expression containing in addition to the glass transition temperatures of the pure components and their concentration the magnitude of the stepwise changes of the specific heat of the pure components at the respective glass transition. The Fox equation results as an approximation if the glass transition temperatures and the specific heat increments are similar for both components of the mixtures. This, of course, is not true in the case considered here. We thus used the more general expression

$\ln T_{\mathrm{g}}=\left(k w_{1} \ln T_{\mathrm{g} 1}+w_{2} \ln T_{\mathrm{g} 2}\right) /\left(k w_{1}+w_{2}\right)$

where $k$ corresponds to the ratio of the specific heat increments $\Delta c_{\mathrm{pi}}$ of the two components at their respective glass transiton and $w_{i}$ to the mass fraction of the components.

The resulting fits are shown in Fig. 4 and the data are given in Tab. 2. We used in this case different values for $k$ ranging from $k=1$ to $k=4$ for PVC as component 1 and the poly(norbornene ester)s as component 2 to take into account the much smaller value of the specific heat increment of the poly(norbornene ester)s. It is obvious that the composition dependence becomes strongly curved and that the extrapolated glass transition temperatures of the pure poly(norbornene ester)s depend very strongly on the value assigned to $k$. 


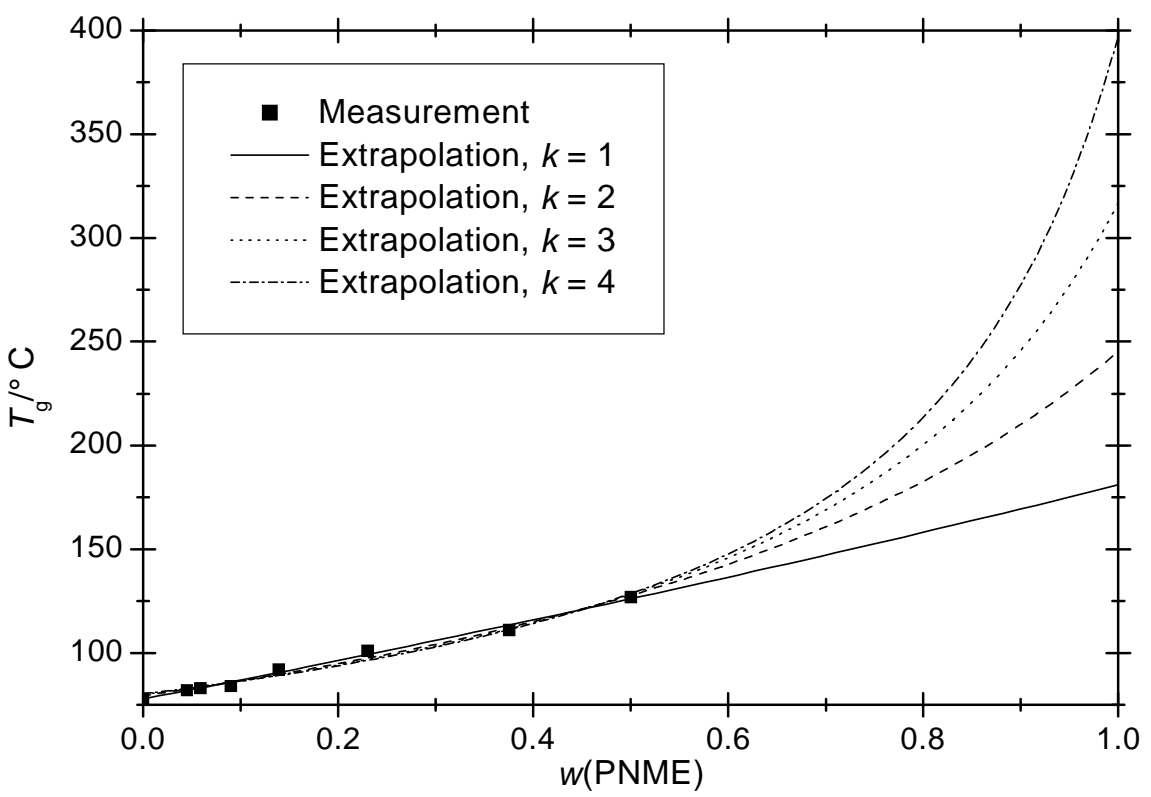

a)

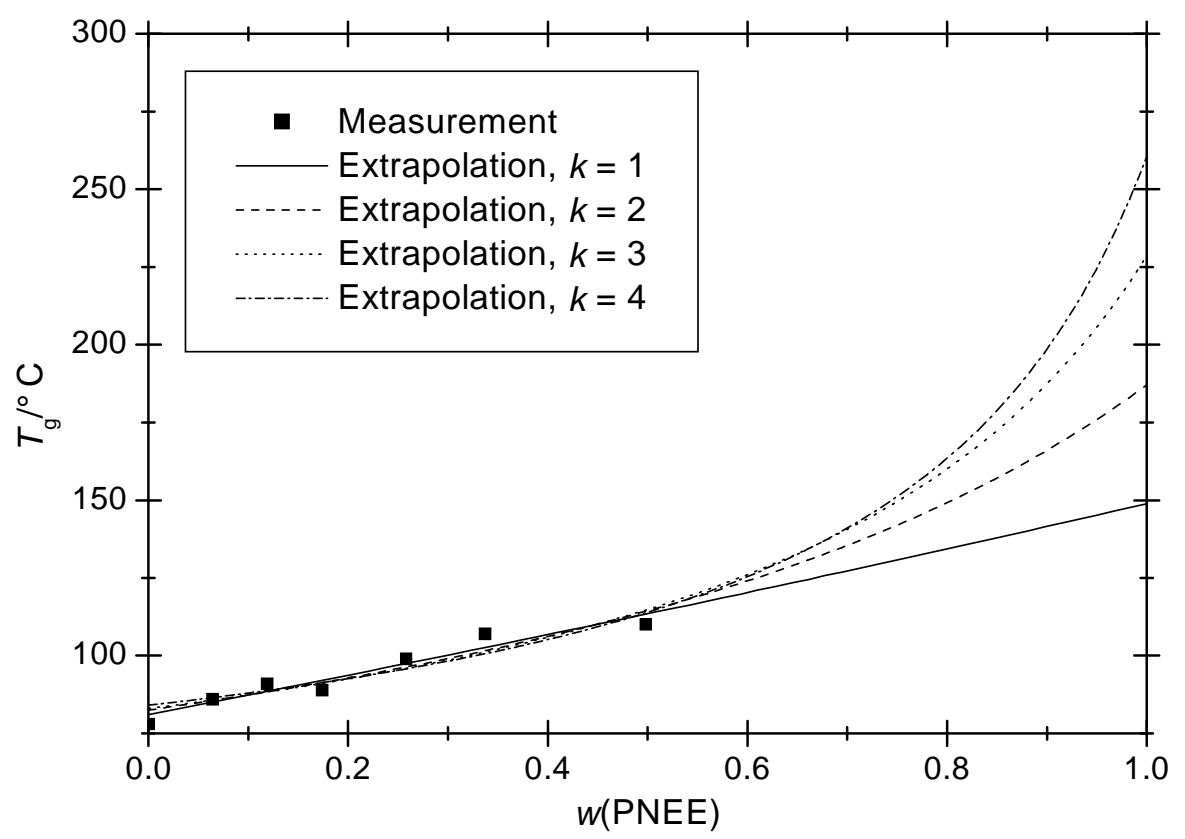

b)

Fig. 4. a) Glass transition temperatures of PNME-PVC blends. b) Glass transition temperatures of PNEE-PVC blends. Extrapolations according to the Couchman equation

The location is roughly in agreement with the one obtained by simulations if we assume $k$ to be of the order of 4 for the purpose of the extrapolation, i.e., the increase of the specific heat is predicted to be about $0.07 \mathrm{~J} /(\mathrm{g} \cdot \mathrm{K})$ or less for the poly(norbornene ester)s. This is very low indeed, yet it fits the results obtained for the blends.

The results reported above show that it can be dangerous to apply the Fox equation to obtain an extrapolated value for the glass transition despite the fact that the fit seems to be very good. In fact, Fig. 3 includes such a plot. The extrapolated glass 
transition temperatures of the esters are very much below the actual values. One, apparently, has to take additional thermodynamic data into account to obtain a good estimate on this value.

Tab. 2. Glass transition temperatures of PNME and PNEE according to the Couchman equation for different values of the specific heat increment ratio $k$

\begin{tabular}{ccc}
$k$ & \multicolumn{2}{c}{$T_{\mathrm{g}} /{ }^{\circ} \mathrm{C}$} \\
\cline { 2 - 3 } & $\mathrm{PNME}$ & PNEE \\
\hline 1 & $181 \pm 3$ & $149 \pm 8$ \\
2 & $245 \pm 8$ & $187 \pm 16$ \\
3 & $317 \pm 16$ & $229 \pm 27$ \\
4 & $397 \pm 23$ & $260 \pm 34$
\end{tabular}

\section{Physical aging studies}

Physical aging involves the relaxation of the non-equilibrium thermodynamic state of the glass towards the equilibrium value of the supercooled melt on annealing within the glassy state [12-16]. Enthalpy and volume relaxation have been studied in detail for flexible chain polymers in the past including PVC. In calorimetric investigations the enthalpy relaxation results in the formation of a peak in the specific heat in the neighbourhood of the glass transition temperature superimposed on the stepwise increase of the specific heat.

Fig. 5 displays thermograms of the pure components and blends with PVC obtained after annealing $15 \mathrm{~K}$ below the glass transition temperatures for about $24 \mathrm{~h}$. PVC and blends containing only small amounts of the poly(norbornene ester) display a well defined aging peak whereas the pure polynorbornene component and blends with higher concentrations of the poly(norbornene ester)s display no such aging peak at all.

The weak tendency towards aging may have two different origins. One is that the dense packing and the low magnitude of the fractional free volume give rise to extremely long relaxation times well beyond the experimental time scale. The other origin may be based on the very small value of the jump of the specific heat of the poly(norbornene ester)s and selective blends with PVC. The temperature dependent variation of the enthalpy does not display, in this case, a significant change in the slope as usually observed for glass forming materials (Fig. 6). This, in turn, should have the consequence that aging effects related to the relaxation of the enthalpy from the nonequilibrium glassy state towards the equilibrium values of the supercooled melt should be weak or absent in the polynorbornene derivatives and in blends with PVC containing higher concentrations of the ester. 


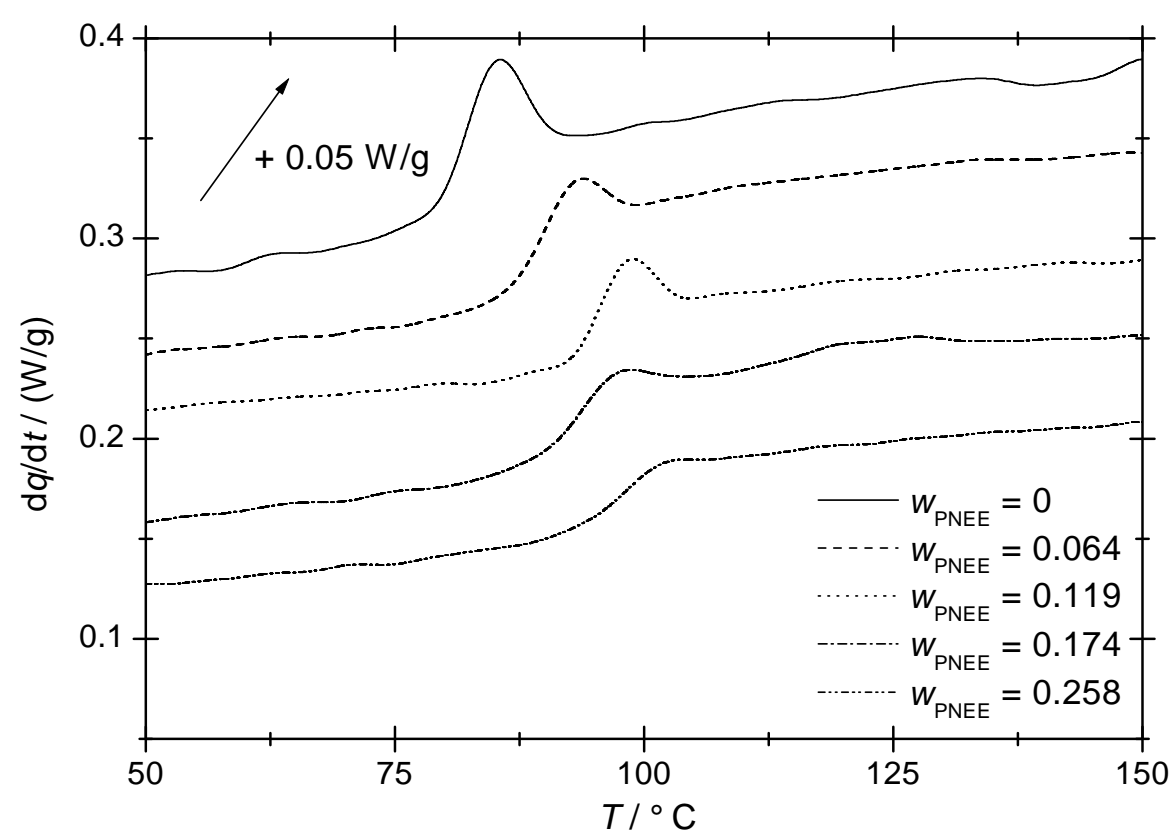

Fig. 5. DSC thermograms of PNEE-PVC blends after annealing for $24 \mathrm{~h}$ at $15^{\circ} \mathrm{C}$ below $T_{\mathrm{g}}$. Curves have been plotted with an offset of $0.05 \mathrm{~W} / \mathrm{g}$ between each curve from below to above

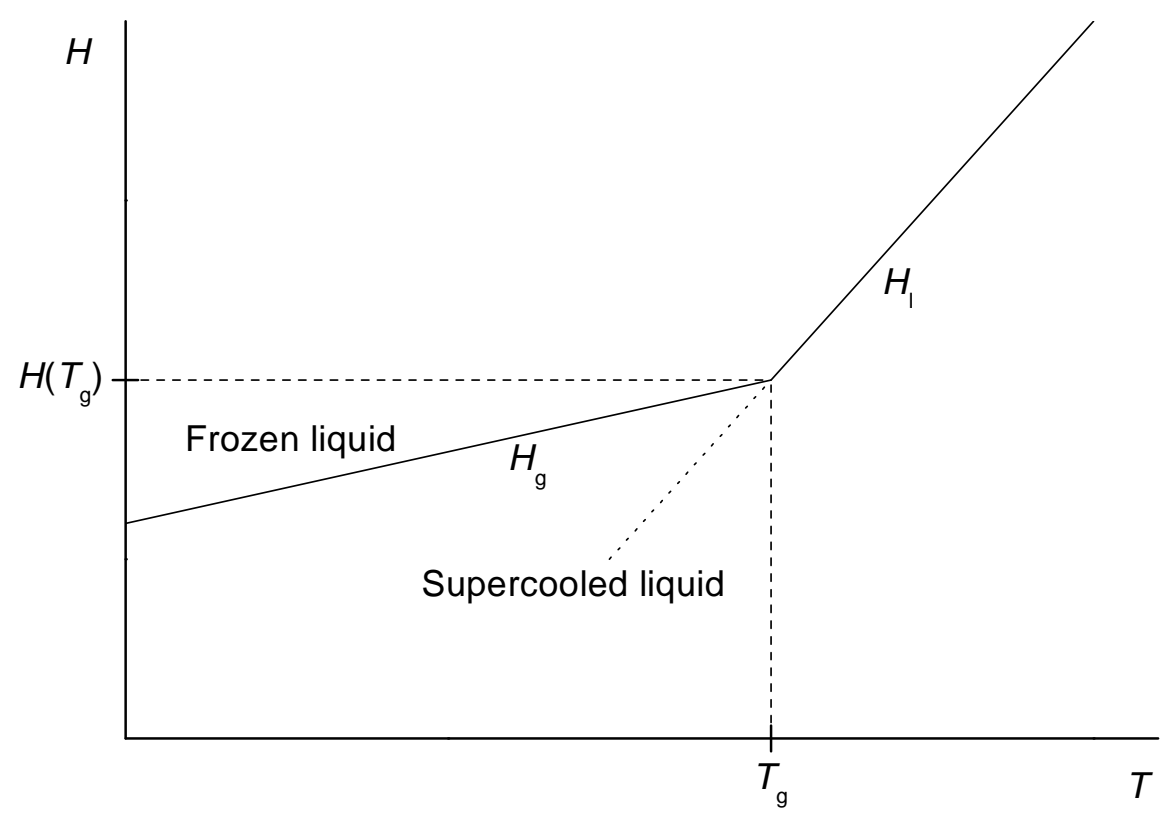

Fig. 6. Temperature variation of the enthalpy at the glass transition (schematic)

\section{Conclusions}

The investigations reported above have revealed that rigid yet coiled chain molecules characterized by strong rotational confinements display unusual freezing-in processes. In polymer blends this unusual property is apparently strongly imposed on the second component even if this component is a flexible chain polymer. Our 
tentative conclusion is that the rigidity of the chain molecules prevents jumps across torsional barriers and the formation of significant amounts of free volume as a function of the temperature even at temperatures well above the glass transition temperature.

Acknowledgement: We would like to acknowledge the financial support by the Deutsche Forschungsgemeinschaft and the Fonds der Chemischen Industrie. Furthermore, we thank Dr. B. S. Heinz and Prof. Dr. W. Heitz for the preparation of the polynorbornene samples.

[1] Haselwander, T.F.; Heitz, W.; Krügel, S.A.; Wendorff, J.H.; Macromol. Chem. Phys. 1996, 197, 3435.

[2] Haselwander, T.F.; Heitz, W.; Krügel, S.A.; Wendorff, J.H.; Macromolecules 1997, 30, 5345 (1997).

[3] Haselwander, T.F.; Heitz, W.; Krügel, S.A.; Raubacher, F.; Wendorff, J.H.; Acta Polym. 1997, 48, 385.

[4] Krügel, S.A.; Raubacher, F.; Wendorff, J.H.; Macromol. Chem. Phys. 1998, 199, 757.

[5] Heitz,W.; Krügel,S.A.; Madan, R.; Wendorff, J.H.; Macromol. Chem. Phys. 1999, $200,338$.

[6] Roth, M.D.; PhD-Thesis, Marburg 2000.

[7] Raubacher, F.; PhD-Thesis, Marburg 2000.

[8] Van Krevelen, D.W.; "Properties of Polymers", Elsevier Pub. Comp., Amsterdam 1972 , p. 34.

[9] Zhao, C.; Rosario Ribeiro, M.; Pinho, M.N.; Subrahmanyam V.S.; Gil, C.L.; de Lima, A.P.; Polymer 2001, 42, 2455.

[10] Mehler, C.; PhD-Thesis, Marburg 1992.

[11] Moro, G.J.; J. Chem. Phys. 1991, 94, 8577.

[12] Moro, G.J.; J. Chem. Phys. 1992, 97, 5749.

[13] Howard, R.N.; "Physics of Glassy Polymers", Haward, R.N., Ed., Wiley, New York, N.Y. 1973.

[14] Roe, R.-J.; Tonelli, A.E.; Macromolecules 1978, 11, 114.

[15] Kovacs, A.J.; Fortschr. Hochpolym.-Forsch. 1964, 3, 394.

[16] Hutchinson, J.M.; Ruddy, M.; J. Polym. Sci. B: 1988, 26, 2341.

[17] Heinz, B.S.; Heitz, W.; Krügel, S.A.; Raubacher, F.; Wendorff, J.H.; Acta Polym. 1977, 48, 385.

[18] Flory, P.J.; Adv. Polym. Sci. 1984, 59, 1.

[19] Rigby, D.; Roe, R.; J. Chem. Phys. 1997, 87, 7285. 
[20] Soldera, A.; Macromol. Symp. 1988, 133, 21.

[21] Mayo, S.L.; Olafson, B.D.; Goddard III, W.A.; J. Phys. Chem. 1990, 94, 8897. [22] Cornell, W.D.; Cieplak, P.; Bayly, C.I.; Kollman, P.A.; J. Am. Chem. Soc. 1993, $115,9620$.

[23] (a) Parinello, M.; Rahman, A.; J. Appl. Physics.1981, 52, 7182 ; (b) Hoover, W.; Phys. Rev. 1985, A31, 1695.

[24] Flory,P.J.; "Statistical Mechanics of Chain Molecules", Hanser Verlag/Gardner Publications Inc. 1988.

[25] Fox, T.G.; Bull. Am. Phys. Soc. 1956, 1, 123.

[26] Gordon, J.M.; Rouse, G.B., Gibbs, J.H., Risen, W.M., J. Chem. Phys. 1997, 66, 4971.

[27] Couchman, P. R.; Karasz, F.E.; Macromolecules 1978, 11, 117.

[28] Couchman, P.R.; Macromolecules 1978, 11, 1156. 1980, 13, 1272. 